\title{
Bioplástico: diversificación de la industrialización de la caña de azúcar que contribuye con el medio ambient
}

Los residuos de embalajes plásticos de origen petroquímico, durante el transcurso de la historia humana se tornaron un problema ambiental, debido a largos años que llevan para su descomposición. De esta forma, ha aumentado las investigaciones sobre la innovación de polímeros biológicos que puedan atender a las necesidades de las personas y al mismo tiempo que reduce los impactos ambientales. En el mundo, la Braskem es una empresa pionera en la producción de polímeros biológicos proveniente del bagazo de caña de azúcar, siendo utilizado en diferentes formatos con finalidades distintas. El objetivo de esta investigación es levantar las actividades de la industria sucroenergética a favor de la producción de polímero biológico. La metodología se trata de un análisis documental, de divulgación hecha por la empresa en su Website a partir de 2011, referente a proveedor de materia prima, beneficios de la producción de bioplástico, el mercado mundial del producto y la influencia en los resultados financieros de la empresa productora. Los resultados identificados retratan que a Braskem ofrece instrucciones a los proveedores, sobre la producción de materia prima que será para la producción de polímero. La lista de siete beneficios vinculados a esta producción, que puede influenciar en la decisión en adquirir este tipo de polímero. Además de eso, la Braskem informa para donde y cuales empresas adquieren sus polímeros, siendo identificadas la comercialización interna y externa en el Brasil y la influencia de la comercialización de polímeros en su ingreso neto expresado en el ingreso porcentual.

Palavras-chave: Innovación; Preservación de los Recursos Naturales; El Plástico Biodegradable.

\section{Bioplastics: diversification of industrialization of cane sugar contributes to the environment}

\begin{abstract}
Waste plastic packaging of petrochemical origin, became in the course of human history an environmental problem because of the number of years that lead to decomposition. Thus, there is growing research on innovation biological polymers that meet the needs of individuals and at the same time reduce environmental impacts. Worldwide, Braskem is a pioneer in the production of biopolymer originating from bagasse of sugarcane, which is used in different packaging formats for different purposes. The research objective is to raise the activities of the sugarcane industry in favor of the production of biopolymer. The methodology comes from the documentary analysis of disclosures made by the company on their website from 2011, relating to: supply of raw materials, benefits of bioplastics production, markets that are marketed products worldwide and the influence of this product the financial results of the production company. The results depict identified Braskem instructs the suppliers on the production of the raw material to be used for the production of the polymer. Lists seven benefits linked to this production, which may influence the decision to acquire this type of polymer. In addition, Braskem announces where and which companies acquire their polymer, identified the internal and external marketing to Brazil and the influence of polymer market in its net income expressed in percentage increase.
\end{abstract}

Keywords: Innovation; Preservation of Natural Resources; Biodegradable Plastic.

\section{Topic: Logística Reversa}

Reviewed anonymously in the process of blind peer

Maycon Jorge Ulisses Saraiva Farinha

Universidade Federal da Grande Dourados, Brasil

http://lattes.cnpq.br/3192437658476559

maycondds@hotmail.com

Luciana Virginia Mario Bernardo

Universidade Estadual do Oeste do Paraná, Brasil

http://lattes.cnpq.br/2732409962619361

lucianamario@yahoo.com.br

Nelson David Lesmo Duarte

Universidad Nacional de Asunción, Paraguai

http://lattes.cnpq.br/4106938744644062

nelsondavlesmd@hotmail.com

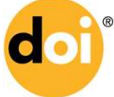

DOI: 10.6008/SPC2179-6858.2016.003.0013
Received: 20/03/2016

Approved: 15/08/2016

Paulo Sérgio Vasconcelos

Universidade Federal do Rio de Janeiro, Brasil

http://lattes.cnpq.br/1981297838530523

paulosvasconcelos@ufgd.edu.br

Referencing this:

FARINHA, M. J. U. S.; BERNARDO, L. V. M.; DUARTE, N. D. L.; VASCONCELOS, P. S.. Bioplástico: diversificación de la industrialización de la caña de azúcar que contribuye con el medio ambiente. Revista Ibero-Americana de Ciências Ambientais, v.7, n.3, p.160-169, 2016. DOI: http://doi.org/10.6008/SPC2179-6858.2016.003.0013 


\section{INTRODUÇÃO}

Las organizaciones, para desarrollar sus actividades productivas de bienes y servicios, deben estar atentas a las externalidades de este proceso. La utilización de los recursos debe estar vinculada a la perspectiva de la sostenibilidad de la producción teniendo en cuenta los aspectos económicos, sociales y ambientales. De esta manera, el ciclo de vida del producto generado debe ser observado por la empresa para que se pueda practicar intervenciones que mejoren los aspectos de sostenibilidad (SLOMSKI et al., 2011).

Para Dias et al. (2013), la necesidad de sostenibilidad de la producción tiene como aliada a la biotecnología, que tiene como característica acciones a favor de la reducción de los impactos ambientales. Las tecnologías producidas por esta ciencia tienen como foco auxiliar las interacciones entre el hombre y la naturaleza, de modo que haya reducción de prácticas no sostenible por medio que envuelve elementos biodegradables.

En la actualidad, la sociedad es sumergida en el uso de embalaje plástico, que se tratan de utensilios producidos, en la mayoría de las veces, a partir de procesos petroquímicos y que pueden ser considerados como externalidad negativa de la industrialización, cuando se observa el impacto en el medio ambiente y su fuente no renovable de materia-prima. Morone et al. (2015) consideran que los factores del aumento de la población mundial y limitados recursos naturales pueden generar una producción menor que la necesidad o estimular el uso de residuos como insumos a otro proceso productivo. En esta perspectiva del uso de residuos, Amulia et al. (2016) observan el creciente interés de los investigadores por los polímeros fabricados a partir de elementos biológicos, debido a la característica de ser biodegradables y versátiles.

Los polímeros, genéricamente identificados por el término de "bioplástico" en algunos sectores de la economía tiene uso representativo, sea para los productos de corta o larga duración como en la agricultura y en la gastronomía (EUROPEAN BIOPLASTICS, 2015). Segundo Telles et al. (2011) e Vista et al. (2015), a partir de uso de insumos renovables, y con mejor proceso de descomposición en la naturaleza, el bioplástico producido en el Brasil es un subproducto de la industrialización de caña de azúcar, debido a su origen en la producción de etanol

En 2008, a Braskem S. A. innovó en la producción de bioplástico en el Brasil. La fabricación en escala fue iniciada en año 2010, con el país se tornó líder en este segmento, con la producción de 200 mil toneladas por año. Desde el inicio de la producción, el bioplástico se destinó a atender a las exigencias de calidad del mercado externo, para diferentes productos y embalaje de distintos sectores (BRASKEM, 2010). El objetivo de la investigación es levantar las actividades de la industria sucroenergetica a favor de la producción de polímero biológico.

\section{REVISIÓN TEÓRICA}

\section{Elementos Biodegradables a Favor de la Preservación Ambiental}

Para Atarés e Chiralt (2016) y Touchaleaume et al. (2016), el descarte indiscriminado del plástico derivado del petróleo es un grave problema para el medio ambiente debido a la contaminación de suelo y 
de las aguas causada por el contacto con el polietileno tradicional. Se tiene, en ese contexto, la necesidad de alternativas que posibiliten el uso de otras materias primas para embalajes plásticos, de modo que estas, por su biodegradabilidad, reducen los impactos en la naturaleza.

Se sabe que el plástico tradicional, no degradable, tiene bajo costo de producción y en su fase liquida puede ser utilizada para la confección de diferentes productos fósiles. La producción en escala mundial aumentó en 243,5 millones de toneladas en el periodo de 1950 a 2008, pues, que la tasa anual de crecimiento fue de $9 \%$. La excelente característica de su versatilidad del plástico fue justamente lo que torno gran problema ambiental, pues, productos descartables pasaron a aumentar la producción y fueron colocados a disposición de los consumidores y, con eso hubo aumento de basura producido en toda parte del mundo (CHANPRATEEP, 2010; PHUKON et al., 2012).

Arrieta et al. (2012) consideran que existen actualmente, muchas inversiones aplicadas a desarrollo del bioplásticos, pues esta tecnología está siendo tomada como una alternativa viable para reducir problemas ambientales oriundo de descarte incorrecto de los plásticos no degradables en la naturaliza. Los bioplásticos, para Chiellini e Solaro (1998), son absorbidos por la naturaleza, pues diferentes microorganismos, como las bacterias, consiguen reducir el ciclo de vida de estos tipos de productos.

Además de eso, Mali et al. (2010), consideran que el bioplásticos son derivados de productos renovables. La materia prima se origina en el sector agrícola, debido a la mayor disponibilidad durante el año y genera un costo menor para para la producción. Chandra et al. (1998) e Telles et al. (2011) afirman que la producción de plástico biodegradable todavía tiene un costo elevado en relación a aquellos considerados no degradables, por eso son necesarias nuevas investigaciones sobre la mejor utilización en diferente tipos de embalajes.

En relación con la comercialización de productos identificados con origen en bioclásticos, Mahanty et al. (2002) concluyen que son aceptados comercialmente, en especial a partir del marketing ecológico ${ }^{1}$. La demanda por los embalajes tiende a crecer y como consecuencia su producción. Ocurre, que según Soroudi y Jakubowicz (2013), la competitividad de este tipo de embalaje, por su alto costo de producción, depende de cómo se mantiene el precio del petróleo, que, si mantiene un precio bajo, su competitividad es prácticamente es imbatible.

En relación al Brasil, Philp et al. (2013) afirman que, a partir de la biomasa de la caña de azúcar, el país tiene condiciones de liderar el mercado de bioplástico. Ocurre que el proceso de innovación es dinámico y necesita ser continuo para que la producción sea perfeccionada y traiga mejores condiciones de costo al productor, como también atienda cada vez mejor a los requerimientos de los consumidores.

\section{El Biolplástico de Origem Sucrialcoholero}

\footnotetext{
${ }^{1}$ Para da Silva et al. (2015a), El marketing ecológico, también conocido como "marketing verde", utiliza de estrategias para llenar las nuevas exigencias del consumidor con relación al medio ambiente y, en contrapartida, objetiva obtener ventajas competitivas con relación a los competidores presentes no mercado.
} 
Para Silva et al. (2013b), el sector sucroalcoholero tiene invertido en la diferenciación de su producción. La biomasa de caña de azúcar, dentro de los otros productos, está siendo utilizada en la biorrefinería como materia prima del bioplástico. Según International Energy Agency - IEA (2010), las biorrefinerías desarrollan actividades a favor la utilización sostenible de biomasa que resulten en productos o energía. El bioplástico, para Stevens (2002) y Rosa et al. (2002), proveen de materia biodegradable, o sea, materias que se desintegra en la naturaliza con facilidad, que son de origen de fuente renovable, sin toxicidad y se integra con el agua debido a sus componentes.

En proceso productivo de bioplástico, Otaviano (2011) indica que, después de la producción de etanol, dicho producto fue utilizado para la construcción de polímeros que, al ser procesados, generan bioplástico. Esta tecnología comenzó a ser desarrollada en los años 1970 por Petrobrás, que consigue extraer sustancia eteno del etanol. La Petrobrás, al aliarse con la Braskem, inició la comercialización del plástico en 2009, después de la innovación del proceso productivo.

Marques (2010) habla de que el Brasil es el mayor productor mundial de caña de azúcar. Así, puede tener el potencial para suplir el aumento de la demanda mundial de bioplástico. Para Lima y Soura (2014), las producciones que se diferencian del etanol y del azúcar en el sector sucroenergetico, como es el caso del plástico verde, contribuyen para que haya nuevos desafíos productivos de materia prima para satisfacer las necesidades del mercado y auxilian en la competitividad del sector.

La Braskem (2012) considera que el bioplástico producido de la caña de azúcar contribuye con la preservación de la naturaleza a través de la reducción de emisión de gas de efecto invernadero, por ser producido a partir de materia- prima totalmente renovable y los embalajes pueden ser reciclados. Además de eso, la eficiencia de desempeño de este bioplástico se iguala al plástico producido a partir del petróleo.

Corroborando este entendimiento de la Braskem, Camo et al. (2012) demuestran que, en el mercado mundial del bioplástico, está siendo, aumentando la aceptación del producto. El bioplástico que dio origen en la caña de azúcar posee características semejantes a los plásticos tradiciones y puede ser recibido en los mismos equipamientos tradicionales y puede ser reciclado en los mismos equipamientos tradicionales de reciclaje. Su diferencial está en el impacto ambiental cuando descartado en la naturaleza impacto mucho menor y mucho menos persistente.

\section{METODOLOGIA}

La investigación fue desarrollada por medio de análisis documental de divulgaciones hechas por Braskem, en su website, a partir de 2011, año inicial de la comercialización de la producción de bioplástico. Los datos fueron recabados en enero de 2016 y trata de los siguientes elementos: proveedor de materia prima, beneficios de la producción de bioplástico, mercados mundiales de los productos y la influencia de este producto en los resultados financieros de la empresa productora.

Los datos están organizados en tablas y gráficos conforme a las necesidades de su presentación y de la argumentación que se pretende. Las discusiones de los resultados fueron realizadas a partir de textos seleccionados en las bases de Science Direct y Scielo. 


\section{RESULTADOS Y DISCUSIÓN}

\section{Requisitos para proveer materias primas a la producción de Bioplástico}

La Braskem considera importante tener, en su producción, elementos de sostenibilidad relacionadas a la ley brasileña. De esta forma, señala a sus proveedores cinco factores centrales y decisivos para la comercialización de materia prima para la fabricación a base de caña de azúcar. Los elementos mencionados se refieren a quema de área agrícola, la biodiversidad, buenas prácticas ambientales, derechos humanos y de trabajadores, y el análisis de los ciclos de vida (BRASKEM, 2015). La tabla 01 trae informaciones combinadas con las especificaciones de cada uno de los elementos:

Tabla 01: Condiciones para proveedores de materia prima al bioplástico de caña de azúcar.

\begin{tabular}{|c|c|}
\hline Elementos & Características \\
\hline Quema & $\begin{array}{l}\text { - Meta para área no mecanizadas, con iniciación superior a 12\% = sin quema hasta } 2017 . \\
\text { - Áreas de expansión de la producción de caña =prohibido quemar para cosechar. } \\
\text { - Quema de bagazo de caña u otro subproducto = debe ser realizado en ambientes cerrados. }\end{array}$ \\
\hline Biodiversidad & $\begin{array}{l}\text { - Mapeamiento de matas ciliares próximas al siembra de caña y proyecto de recuperación y } \\
\text { preservación de matas ciliares en todas las propiedades } \\
\text { - Preservación de nacientes de agua y matas ciliares en relación las mismas, con radio mínimo de } 50 \\
\text { metros. } \\
\text { - Promoción de actividades con intuito de regenerar áreas de preservación permanente cerca de las } \\
\text { usinas. } \\
\text { - Usar medidas que previenen incendios en las matas ciliares, como: brigada de combate al fuego, } \\
\text { como carro equipado y acero (remoción en lo mínimo } 6 \text { metros de vegetación entre áreas cultivada } \\
\text { y la mata ciliar). } \\
\text { - Utilizar instrucciones de zonificación agroecológica de caña de azúcar, del Ministério da Agricultura, } \\
\text { Pecuária e Abastecimento, en vigencia. } \\
\text { - No cultivar caña de azúcar en áreas de los Biomas: Amazonia y Pantanal y en la Bacía del Alto } \\
\text { Paraguay. } \\
\text { - Preservar áreas de protección ambiental o aquellas que las substituyen. }\end{array}$ \\
\hline $\begin{array}{l}\text { Buenas Prácticas } \\
\text { Ambientales }\end{array}$ & $\begin{array}{l}\text { - Descarte correcto de embalajes de defensivos agrícolas. } \\
\text { - En el proceso industrial, utilizar acciones que promuevan la reducción de la polución atmosférica. } \\
\text { - Mantener prácticas de reciclaje y reutilización de residuos oriundos de la producción de etanol. } \\
\text { - Utilizar acciones que conserven el suelo y reduzcan las erosiones. } \\
\text { - Conservación de los recursos hídricos, reutilización del agua para captar mínimamente este } \\
\text { recurso, tener plan de acción a partir de metas de reducción de uso da agua por tonelada de caña } \\
\text { procesada. } \\
\text { - Áreas sin la necesidad de irrigación deben ser priorizadas para el cultivo de caña. } \\
\text { - Uso de control biológico de plagas. } \\
\text { - Priorizar prácticas de salud, seguridad y medio ambiente. }\end{array}$ \\
\hline $\begin{array}{l}\text { Derechos } \\
\text { Humanos y } \\
\text { Trabajadores }\end{array}$ & $\begin{array}{l}\text { - Respeto a los derechos humanos reconocidos internacionalmente. } \\
\text { - Registro en cartera de trabajo y Previsión Social de todos los funcionarios. } \\
\text { - Respetar acuerdos y normas colectivas de trabajos. } \\
\text { - Disponibilidad de alojamiento con calidad para trabajadores migrantes. } \\
\text { - Mantener comisión interna de prevención de accidentes en el trabajo rural. } \\
\text { - Distribuir gratuitamente equipamientos de protección individual para todos los trabajadores. } \\
\text { - Ofrecer transporte seguro a los trabajadores. } \\
\text { - Ofrecer equipos térmicos higiénicos y que mantengan los alimentos calientes. }\end{array}$ \\
\hline $\begin{array}{l}\text { Análisis del Ciclo } \\
\text { de Vida do } \\
\text { Producto }\end{array}$ & $\begin{array}{l}\text { - Brindar informaciones como: consumo de energía de todo el proceso productivo, incluyendo } \\
\text { transporte; consumo de recursos naturales; uso de la tierra; residuo; potencial de toxicidad humana } \\
\text { y potencial de riesgos y enfermedades ocupacionales. }\end{array}$ \\
\hline
\end{tabular}

Fuente: Elaboración a partir de Braskem (2015a). 
Estas especificaciones marcadas en el tabla de arriba pueden contribuir para la mejoría del sector sucroenergético brasileiro y la producción, considerando las inversiones para la diversificación de productos y subproductos derivados del proceso de innovación de las organizaciones (SILVA et al., 2013b). Además, Marquez (2010) confirma que Brasil tiene una producción exponencial de la materia prima de bioplástico este producto, como ya dicho, tiene una diferencia con los demás debido a la característica biodegradable, o sea, reduce los impactos ambientales cuando se desarrolla en la naturaleza. De ese modo puede ser favorable a la sostenibilidad, con eso, las organizaciones envueltas en el proceso productivo deben actuar contemplando, es sus acciones, los aspectos económicos, sociales y ambientales, tal como fueron incluidas por la Braskem (2015a) en sus normas para los proveedores de la materia prima.

Para Chiellini y Solaro (1998), la reducción del ciclo de vida del plástico es una de las características de los bioplástico. Así, el análisis de ciclo es necesario para que se tengan informaciones que posibiliten la reducción de los impactos ambientales negativos. De esta forma, para que se tengan informaciones de toda la cadena productiva del bioplástico, los proveedores deben contribuir disponibilizando informaciones necesarias

\section{Beneficios de la producción de bioplástico de caña de azúcar y los sectores que sus productos atienden}

Con relación a los beneficios de la producción de bioplástico, la Breskem (2015b) hace una lista de siete factores que favorecen la producción de bioplástico a partir de la caña de azúcar, organizados conforme a la proximidad del asunto. La producción de la materia prima, la producción por área, sus respectivos aumentos y adaptación a las altas temperaturas son cuatro ventajas de esta materia prima. Según Lima y Souza (2014), caso que haya expansión en el portafolio de productos o demandas de aquellas ya existentes, hay tendencia del aumento productivo de la caña para atender las diferentes necesidades productivas. Para Mahanty et al. (2002), el bioplástico tiene aceptación comercial debido a su relación con marketing verde y tiende al crecimiento de consumo.

Dado que el bioplástico es un producto vinculado a la producción de etanol, entonces, en relación con ese factor de utilización de residuos de producción, Morone et al. (2015) argumentan que el uso de residuos como insumo es un proceso natural, influenciado por el aumento poblacional y por la finitud de los recursos naturales. El costo de la producción del etanol de caña de azúcar es considerado $25 \%$ del costo de otras materias primas que son utilizados con la misma finalidad. El costo es un factor considerado en esta producción debido a lo indicado por Chandra y Rusthi (1988) y por Telles et al (2011), de que los plásticos biodegradables pueden tener un costo superior a aquellas no degradables.

La producción de materia prima de caña puede también contribuir con aspectos ambientales con el uso de áreas degradadas para la expansión y utilizar menos fertilizantes y defensivos, debido a la duración de 5 años de ciclo productivo. Además de eso, el IEA (2010) agrega que las biorrefinerías actúan en vinculación a la sostenibilidad del uso de las biomasas. En esta perspectiva del uso de residuos, se trata de una innovación al sector. Amulia et al. (2016) observan el crecimiento interesante de los investigadores por 
los polímeros criados a partir de elementos biológicos, no solamente por su carácter de biodegradabilidad, además por la versatilidad de uso de este material para satisfacer nuevas demandas del mercado.

La producción de plástico verde atiende al sector de alimentación humana, de animales y de otros sectores de la vida de la población como: utensilios domésticos, maquinarias de lavarropas, basureros, material de escritorio, protector solar, papel higiénico, productos de belleza, cascos industriales, bolsa de plásticos, granos y forrajes, herramientas y equipos para jardín, agricultura y construcción civil. Para Arrieta et al. (2012), el bioplástico es una alternativa para reducir los problemas ambientales generados por el plástico no biodegradable y es así que la diversificación del uso del plástico contribuye con esa reducción. Las empresas consumidoras de embalajes a partir de plástico verde están localizadas en diferentes países, como registrado en la Tabla 02.

Tabla 02: Localización de empresas consumidoras de bioplástico.

\begin{tabular}{|l|l|l|}
\hline Continente & País & Tipo de Producto \\
\hline \multirow{3}{*}{$\begin{array}{l}\text { América del } \\
\text { Uur }\end{array}$} & Brasil & $\begin{array}{l}\text { Frascos para cuidado personales; Embalajes rígidas; Bobinas Técnicas para alimentación } \\
\text { humana; Embalajes para alimentación animal. }\end{array}$ \\
\cline { 2 - 3 } & Argentina & Embalaje para cuidados domésticos; Bisnaga para cuidado personal. \\
\cline { 2 - 3 } & Uruguay & Frascos para cuidado personal; \\
\hline $\begin{array}{l}\text { América do } \\
\text { Norte }\end{array}$ & $\begin{array}{l}\text { Estados } \\
\text { Unidos }\end{array}$ & Frasco para cuidado doméstico; Película para alimentación humana. \\
\hline Europa & & Bolsas duraderos; Coating y Tapas para bebidas; Tapón para bebidas; Basureros. \\
\hline Asia & Corea del Sur & Aplicaciones. \\
\hline & Japón & Embalajes para cuidado personal. \\
\hline Oceanía & & Frascos para cuidado personal. \\
\hline
\end{tabular}

Fuente: Elaboración a partir de Braskem (2016).

Continentes como Europa y Oceanía no tienen disponibles los países donde se comercializa el bioplástico y también no existe justificativa por la falta de información. América del Sur es el continente con mayor número de países atendidos, bien con mayor diversificación de productos comercializados. Se explica, con la tabla, que la empresa productora de bioplástico atiende a diferentes necesidades del mercado.

Para Carmo et al. (2012), el mercado mundial está más accesible a la aceptación de bioplástico, principalmente el plástico de origen de caña de azúcar, esto es debido en especial a las semejanzas al plástico tradicional, hasta mismo en el uso de los mismos equipamientos de reciclaje. Además de eso, son considerados, obviamente, los beneficios presentado por Stevens (2002) y Rosa et al. (2002) sobre o bioplástico y su integración con la naturaleza.

\section{La influencia del plástico en los resultados financieros de la Braskem}

La producción del bioplástico de caña de azúcar, como menciona Otaviano (2011), es el resultado de la unión entre la Braskem y la Petrobras. Su proceso productivo fue iniciado en 2009, y las primeras informaciones sobre la comercialización comprenden el periodo de 2011 y 2014, como se indica en el Grafico 01.

El grafico indica el crecimiento positivo de la población del bioplástico brasilero y de su contribución con el lucro liquida de la empresa, en que el mayor crecimiento ocurre en el periodo de 2011 a 2012. Con relación a la contribución al Lucro liquido de la Braskem, en que cuatro años el bioplástico es responsable 
por un tercio del lucro anual. Para Arrieta et al. (2012), el bioplástico necesita de muchas inversiones en tecnología para conseguir la producción, sin embargo, la contribución en el lucro liquido demuestra que este producto puede influenciar en los resultados de la organización. Además, están disponible informaciones sobre margen operacional del bioplástico, como consta en la tabla 03.

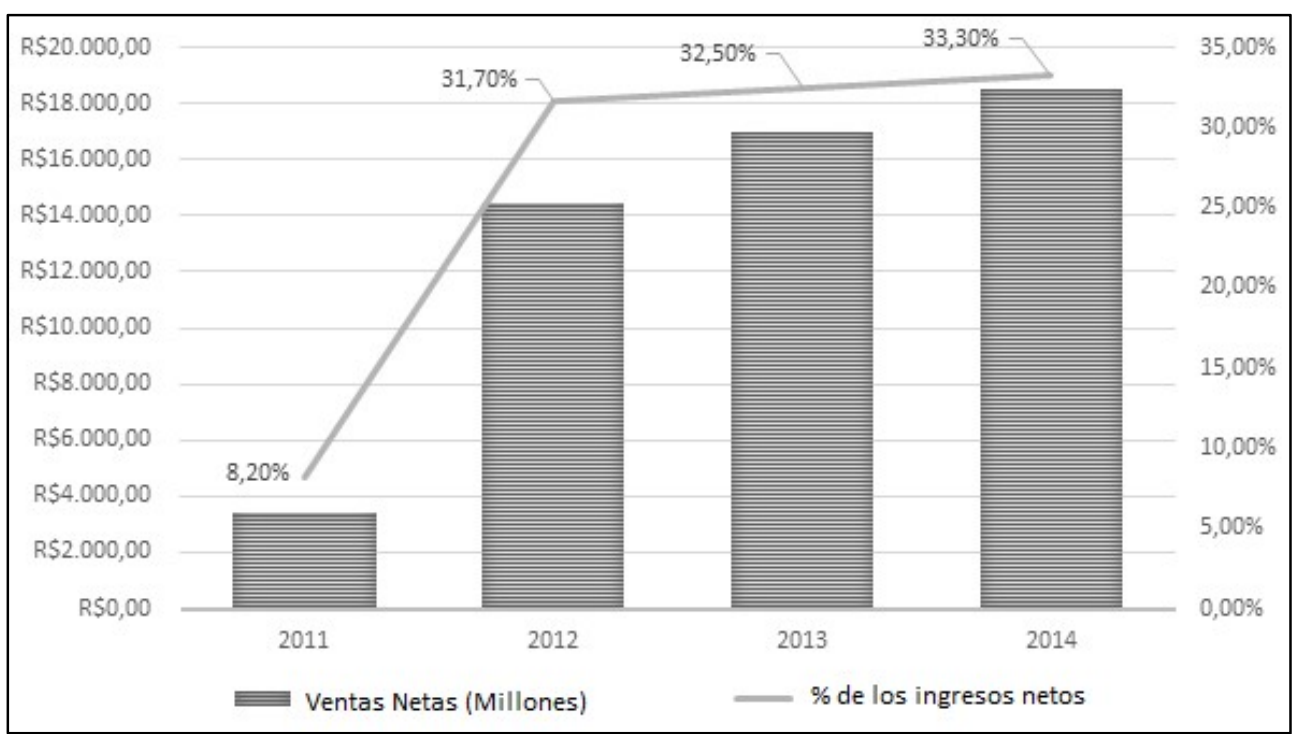

Gráfico 01: Comercialización de la producción y porcentaje de contribución en Lucro Liquida de la empresa. Fuente: Elaborado a partir de los reportes de la Administración de 2011 a 2014.

Tabla 03: Relación año y Margen operacional del Bioplástico

\begin{tabular}{ccc}
\hline Período & Margen Operacional (\%) & Diferencia Porcentual entre Anos (\%) \\
\hline 2011 & $(0,4)$ & -- \\
2012 & 3,0 & 3,4 \\
2013 & 8,1 & 5,1 \\
2014 & 10,2 & 2,1 \\
\hline
\end{tabular}

Fuente: Elaborado a partir de los reportes de la Administración de 2011 a 2014.

Con relación a la margen operacional, o sea, al lucro de la producción de bioplástico antes de deducciones (como impuesto de renta y de la distribución a los financiadores de las actividades del negocio), se observa que, en cuatro años, alcanzo 10,6\%. Así, cuanto mayor está el porcentaje, mejor para la organización. Tuvo su mayor porcentaje en el periodo de 2012 a 2013.

Resultados de la comercialización de la producción de bioplástico, Philp et al. (2013) demuestran las condiciones positivas del Brasil en liderar el sector. Para eso, el proceso de innovación de la producción debe ser continuo y corresponder con las necesidades del mercado, en virtud de la reducción de costos al productor y atendimiento de las necesidades de los consumidores con precios semejantes a la producción de plástico no biodegradable.

\section{CONSIDERACIONES FINALES}

La fabricación del plástico biodegradable brasileño es el resultado de un proceso de innovación iniciada en los años 1970 por una empresa del país y mejorado con la cooperación de empresa privada. Diferentes materias primas son probadas y utilizadas para la confección de este producto, siendo una de las más eficaces la caña de azúcar, de que se aproveche la biomasa. Inversiones en la producción pueden ser la 
solución para algunos problemas ambientales ya aceradas en varias partes del mundo, como la acumulación de basura plástica, la descomposición lenta de este material y los impactos ambientales en los suelos y en las aguas, inclusive en las aguas marinas.

El objetivo de esta investigación, entonces, levantar las actividades de la industria sucroenergética a favor de la producción del plástico verde. Analizados los datos obtenidos, se percibe que ya existen orientaciones técnicas para todo el proceso, desde la producción de la quema de biomasa producida en el campo, pasando por los proveedores de materia prima a las industrias, hasta el tratamiento para la producción del plástico. También se pueden notar los diferentes productos que son comercializados mundialmente y cuanto amplio es el mercado, lo que indica que el bioplástico de caña de puede sustituir al plástico tradicional en diferentes situaciones y además genera beneficios a los productores y consumidores.

De esta forma, se considera que invertir en esta cadena productiva y en las tecnologías necesarias para las mejorías en proceso productivo del bioplástico podrá proporcionar ganancias a los diferentes stakeholders y, principalmente, a preservación ambiental de diferentes biomas presentes no mundo.

\section{REFERENCIAS}

ARRIETA, M. P.. Pirólisis de resíduos de bioplásticos: productos obtenidos de ácido poliláctico (PLA). Federación de Asociaciones de Ingenieros Industriales de España (FAIIE). 2012.

ATARÉS, L.; CHIRALT, A.. Essential oils as additives in biodegradable films and coatings for active food packaging. Trends in Food Science \& Technology, p.51-62, 2016.

AMULIA, K.. Wastewater as renewable feedstock for bioplastics prodution: understanding the role of reactor microenvironment and system $\mathrm{pH}$. Journal of Cleaner Production, v.112, p.4618-4627, 2016.

BRASKEM. Polietileno verde. 2010.

BRASKEM. Biopolímero polietileno verde: inovação transformando plástico em sustentabilidade. 2012.

BRASKEM. Código de conduta para fornecedores de etanol. 2015b.

BRASKEM. Plástico verde: do Brasil para o Mundo. 2016.

BRASKEM. Relatório da Administração: informações referentes a 2011. 2012.

BRASKEM. Relatório da Administração: informações referentes a 2012. 2013.

BRASKEM. Relatório da Administração: informações referentes a 2013. 2014.

BRASKEM. Relatório da Administração: informações referentes a 2014. 2015.

CARMO, R. W.. Polietileno Verde. Boletim de Tecnologia e Desenvolvimento de Embalagens, v.24, n.1, 2012.
CHANDRA, R.; RUSTGI, R.. Biodegradable Polymers. Progress in Polymer Science, v.23, p.1273-1335, 1998.

CHANPRATEEP, S.. Current trends in biodegradable polyhydroxyalkanoates. Journal of Bioscience and Bioengieneering, p.621-632, 2010.

CHIELLINI, E.; SOLARO, R.. Environmentally degradable polymers and plastics. An Overview. In: INTERNATIONAL WORKSHOP ON ENVIRONMENTALLY DEGRADABLE AND RECYCLABLE POLYMERS IN LATIN AMERICAN. Proceedings. Campinas, 1998.

DIAS, B. P.; ROWE, R. V. A.. Bagaço de cana de açúcar: matéria-prima para a fabricação de materiais biodegradáveis. Bioenergia em Revista: Diálogos, v.3, n.1, p.73-87, 2013.

EUROPEAN BIOPLASTICS. Frequently asked questions on Bioplastics. 2015.

IEA. International Energy Agency. Sustainable Production of Second-Generation Biofuels. 2010.

LIMA, N. C.; SOUZA, G. H. S.. A demanda do etanol e sua caracterização no mercado brasileiro de combustíveis. Organizações Rurais e Agroindustriais, v.16, n.4, p.532-544, 2014.

MOHANTY, A. K.. Sustainable bio-composites from renewable resources: opportunities and challenges in the greenmaterials world. Journal of Polymers and the Environment, v.10, 2002.

MALI, S.. Filmes de amido: produção, propriedades e potencial de utilização. Ciências Agrárias, v.31, n.1, p.137156, 2010. 
MARQUES, J. J.. O "plástico verde" e o mercado brasileiro de etanol. Dissertação (Mestrado em Administração) Universidade de São Paulo, São Paulo, 2010.

MORONE, P.. Assessing the potential of biowaste for bioplastics production through social network analysis. Journal of Cleaner Production, v.90, p.43-54, 2015

OTAVIANO, C.. Mudança de petróleo para biomassa impulsiona a química verde com ciência. Revista Eletrônica de Jornalismo Científico, 2011.

PHILP, J. C.. Bioplastic science from a policy vantage point. New Biotechnology, v.30, n.6, 2013.

PHUKON, P.. Bio-plastic (P-3HB-co-3HV) from Bacillus circulans (MTCC 8167) and its biodegradation. Colloids and Surgaces B: Biointerfaces, v.92, p.30-34, 2012.

ROSA, D. S.. Avaliação da biodegradação de poli- $\beta$ (hidroxibutirato), poli- $\beta$ - (hidroxibutirato-covalerato) e poli-î(caprolactona) em solo compostado. Polímeros: Ciência e Tecnologia, v.12, n.4, p.311-317, 2002.

SILVA, A. A.. Inovando a organização pela estratégia de marketing verde. Revista Internacional de Investigación em Ciencias Sociales, v.11, n.2, p.165-178, 2015a.
SILVA, A. T. B.. Cenários prospectivos para o comércio internacional de etanol em 2020. Revista Administração, v. 48, n. 4, p.727-738, 2013b.

SLOMSKI, V.. Sustentabilidade nas organizações: a internalização dos gastos com o descarte do produto e ou embalagem aos custos de produção. Revista Administração, v.47, n.2, p.275-289, 2012.

SOROUDI, A.; JAKUBOWICZ, I.. Recycling of bioplastics, their blends and biocomposites: A review. European Polymer Journal, v.49, p.2839-2858, 2013.

STEVENS, E. S.. Green plastics: an introduction to the new science of biodegradable plastics. Woodstock: Princeton University Press, 2002.

TELLES, M. R.. Produção, propriedades e aplicações de bioplástico obtido a partir da cana-de-açúcar. Ciência \& Tecnologia: FATEC - JB, Jaboticabal, v.2, n.1, p.52-63, 2011.

TOUCHALEAUME, F.. Performance and environmental impacto of biodegradable polymers as agricultural mulching films. Chemosphere, v.144, p.433-439, 2016.

VISTA, H. A. B.. Produto sustentável: equipamento de proteção individual fabricado com plástico verde. Revista de Gestão Ambiental e Sustentabilidade, v.4, n.1, 2015. 